\title{
Transtornos mentais comuns na prática clínica
}

\section{Common mental disorders in clinical practice}

\author{
André Russowsky Brunoni ${ }^{1}$
}

Brunoni AR. Transtornos mentais comuns na prática clínica. Rev Med (São Paulo). 2008 out.-dez.;87(4):251-63.

\begin{abstract}
RESUMO: Histórico: A prevalência de transtornos mentais comuns na população e nos ambulatórios gerais e de especialidade é elevada, o que faz com que os médicos clínicos sejam os principais responsáveis pela identificação, triagem, tratamento e encaminhamento de grande parte dos pacientes portadores de transtornos psiquiátricos. Desta maneira, o conhecimento destas condições pelo médico clínico é essencial no diagnóstico e tratamento dos transtornos mentais. Objetivo: O objetivo deste artigo é rever os principais tópicos relacionados a três transtornos mentais comuns na prática clínica - os transtornos de humor, os transtornos de ansiedade e os transtornos somatoformes e que são os mais prevalentes na cidade de São Paulo. Resultados: O rastreamento dos transtornos depressivos (prevalência ao longo da vida: $17 \%$ ) deve ser feito em qualquer consulta clínica através do questionamento de anedonia e humor deprimido. Os transtornos afetivos bipolares (3-5\%) devem ser descartados antes do diagnóstico de depressão unipolar, uma vez que o erro diagnóstico pode levar a um aumento da gravidade do quadro. Os transtornos de humor, bem como os transtornos ansiosos (8\%), podem ser atualmente manejados de forma bastante segura com vários tipos de antidepressivos. Por outro lado, os transtornos somatoformes (6\%) exigem um acompanhamento conjunto com um especialista - neste caso, cabe ao clínico reconhecer o transtorno e investigar as queixas físicas. Conclusão: $\mathrm{O}$ tratamento de muitos transtornos psiquiátricos pelo médico clínico é factível e deve ser incorporado na sua prática clínica diária, bem como o pronto reconhecimento de transtornos mais graves em que seja necessário o encaminhamento para um psiquiatra.
\end{abstract}

DESCRITORES: Transtornos mentais. Transtorno bipolar. Transtornos somatoformes. Transtornos do humor.

A prevalência de transtornos psiquiátricos nos adultos é alta. Em um estudo mundial realizado em 14 países com 60 mil adultos observou-se que a prevalência anual de qualquer transtorno psiquiátrico é por volta de $20 \%{ }^{1}$. No Brasil, em um estudo epidemiológico realizado em 1997 em três capitais brasileiras (Brasília, São Paulo e Porto Alegre) observou-se que a prevalência ao longo da vida de transtornos psiquiátricos pode chegar a $50 \%{ }^{2}$. Em um estudo de coorte em Bambuí (MG), a prevalência-ano de depressão na população foi de $10 \%{ }^{3}$. Em um estudo realizado recentemente na cidade de São Paulo, mostrou-se que a prevalência de qualquer transtorno psiquiátrico ao longo da vida é de $45 \%$ - mesmo excluindo-se a dependência de nicotina, quase um terço da população apresentará

\footnotetext{
1. Centro de Pesquisa Clínica, Hospital Universitário, Universidade de São Paulo, Brasil. Departamento de Psiquiatria, Faculdade de Medicina da Universidade de São Paulo.
}

Endereço para correspondência: André Russowsky Brunoni. Alameda Fernão Cardim, 346 Apt. 101, São Paulo, SP. E-mail: brunowsky@gmail.com 
algum diagnostico psiquiátrico ao longo da vida ${ }^{4}$.

$\mathrm{Na}$ cidade de São Paulo, os diagnósticos psiquiátricos mais comuns foram: dependência de nicotina ( $25 \%$ ao longo da vida), qualquer transtorno do humor (18,5\%), qualquer transtorno ansioso (12,5\%), transtornos somatoformes (6\%) e dependência de álcool $(5,5 \%)^{4}$. É importante ressaltar que tais condições estão associadas a uma alta morbimortalidade: os hábitos de fumar e ingerir bebidas alcoólicas, por exemplo, estão relacionados com as principais causas de óbito no mundo ${ }^{5}$, enquanto que o transtorno depressivo é uma das principais causas de absenteísmo e comprometimento da qualidade de vida ${ }^{6}$.

Contudo, é freqüente que esses transtornos não sejam reconhecidos na atenção à saúde, na ausência de um médico psiquiatra ${ }^{4,7,8}$. Por isso, é necessário que o médico clínico esteja capacitado, dentro da sua formação geral, a reconhecer, diagnosticar e tratar esses transtornos. O objetivo deste artigo é rever os principais transtornos psiquiátricos na prática clínica, com base nos dados de prevalência na cidade de São Paulo ${ }^{4}$, focando nos critérios diagnósticos e nas principais alternativas de tratamento farmacológico e não-farmacológico. Como, usualmente, o tratamento do tabagismo e do etilismo é multidisciplinar e envolve outras condições e co-morbidades clínicas importantes que estão além do escopo desta revisão, focaremos este artigo nos transtornos do humor, nos transtornos ansiosos e nos transtornos somatoformes. A Tabela 1 lista a prevalência dos transtornos psiquiátricos na cidade de São Paulo.

TABELA 1. Prevalência dos principais transtornos psiquiátricos em São Paulo

\begin{tabular}{lcc}
\hline & Transtorno mental ao longo da vida & Transtorno mental no último ano \\
\hline Qualquer transtorno & $45 \%$ & $25 \%$ \\
\hline Tr. Humor & $15 \%$ & $5 \%$ \\
\hline Tr. Depressivo Maior & $13,5 \%$ & $4,5 \%$ \\
\hline Distimia & $3,5 \%$ & $1 \%$ \\
\hline Tr. Ansiedade Generalizada & $8,2 \%$ & $4 \%$ \\
\hline Dep. Substâncias & $33 \%$ & $18 \%$ \\
\hline Dep. Nicotina & $30 \%$ & $12 \%$ \\
\hline Dep. Alcool & $8 \%$ & $6,5 \%$ \\
\hline
\end{tabular}

Adaptado de Andrade et al., 2002.

\section{Transtornos de humor}

Os transtornos de humor são quadros psiquiátricos caracterizados por alterações acentuadas e razoavelmente bem delimitadas do humor, da cognição, da atenção, dos ritmos biológicos e da psicomotricidade ${ }^{9,10}$. Podem ser divididos em dois grandes grupos: o grupo dos transtornos depressivos unipolares e o dos Transtornos Afetivos Bipolares (TAB), quando os episódios depressivos alternam-se com episódios de mania (TAB tipo I) ou hipomania (TAB tipo II) ou, ainda, quando os sintomas de mania e depressão apresentam-se simultaneamente (Estado Misto).
Os transtornos depressivos unipolares podem ser subdivididos em duas grandes entidades nosológicas: os Transtornos Depressivos Maiores e os Transtornos Distímicos. Os pacientes com distimia apresentam-se, em relação àqueles com Transtornos Depressivos Maiores, com sintomas mais brandos, porém mais crônicos.

Transtornos depressivos unipolares

\section{Diagnóstico}

Os Transtornos Depressivos Maiores e a 
Distimia são condições freqüentes na população em geral e no ambulatórios de clínica médica. Ambos acometem duas a três vezes mais mulheres do que homens, mais comumente a partir da $3^{a}$ década de vida ${ }^{10}$. Os critérios diagnósticos principais são a presença de anedonia e/ou humor deprimido. Outros sintomas importantes são encontrados nos campos cognitivos (pensamentos de culpa, morte, menos valia, dificuldade de concentração e ansiedade) e somáticos (insônia, perda de peso e retardo psicomotor). Os critérios diagnósticos estão listados na Tabela 2 - ao menos cinco desses critérios devem estar presentes por mais de duas semanas para que o diagnóstico seja confirmado.

TABELA 2. Critérios diagnósticos para o transtorno depressivo maior - adaptado de DSM-IV

\begin{tabular}{ll}
\hline Perda de interesse ou prazer & Fadiga ou Perda de Energia \\
\hline Humor deprimido & Sentimento de inutilidade ou culpa \\
\hline Perda ou ganho de peso & Indecisão ou falta de concentração \\
\hline Insônia ou Excesso de sono & Pensamentos de morte \\
\hline Agitação ou Retardo Psicomotor & \\
\hline
\end{tabular}

Pela alta prevalência na população, o rastreamento do transtorno depressivo deve ser ativo e realizado pelo médico geral ${ }^{11}$ - de fato, a força-tarefa de rastreamento norte-americana defende que este procedimento seja adotado em qualquer prática clínica que possa garantir tratamento e seguimento adequados ${ }^{12}$. Vários instrumentos diagnósticos podem ser utilizados, contudo, uma triagem simples e custo-efetiva é questionar o paciente se nas duas últimas semanas, apresentou-se na maior parte dos dias $^{1}$ mais triste do que o habitual ou ${ }^{2}$ com pouco interesse, vontade ou disposição em realizar suas atividades habituais. Se ele responder sim a uma das perguntas, então uma investigação completa deve ser realizada ${ }^{7}$.

\section{Diagnóstico diferencial}

O diagnóstico diferencial dos transtornos depressivos deve ser realizado em relação a outras condições clínicas e psiquiátricas. Há várias doenças clínicas que podem se apresentar com sintomas psiquiátricos, sendo as mais comuns, o hipotireoidismo, a síndrome de Cushing, a síndrome de Addison, o diabetes melito, a AIDS, a anemia, os acidentes vasculares cerebrais e as epilepsias de lobo temporal e outras. Medicamentos como betabloqueadores e outros anti-hipertensivos, corticosteróides, anticoncepcionais orais e quimioterápicos também podem induzir efeitos colaterais que se confundem com sintomas depressivos ${ }^{9}$.
Dentre os transtornos psiquiátricos, os transtornos de ansiedade, obsessivo-compulsivo, de personalidade e somatoformes podem se manifestar por meio de sintomas depressivos, tornando o diagnóstico diferencial difícil e muitas vezes exigindo a avaliação de um psiquiatra.

Ainda, cabe ressaltar que o principal diagnóstico diferencial da depressão unipolar é a depressão bipolar, ou seja, um episódio depressivo atual em um paciente que já tenha apresentado ou possa vir a apresentar episódios de mania ou hipomania. De fato, estudos de seguimento mostraram que cerca de um terço dos pacientes bipolares em episódio depressivo foram erroneamente diagnosticados como deprimidos unipolares ${ }^{13}$ e que pacientes bipolares procuram consulta médica cinco vezes mais quando estão deprimidos do que quando estão em mania ou hipomania ${ }^{14}$. Um diagnóstico incorreto pode fazer com a que a depressão se torne crônica, havendo piora dos sintomas e aumentando o risco de hospitalização e suicídio ${ }^{13}$. Para realizar o diagnóstico diferencial, o clínico deve procurar indícios de bipolaridade. Além de, obviamente, questionar quanto a episódios prévios de mania ou hipomania, existem outros indícios que podem sugerir uma depressão bipolar, sendo os mais importantes a presença de antecedentes familiares de Transtornos Afetivos Bipolares e de "virada hipomaníaca" (ou seja, a apresentação de sintomas de mania ou hipomania após o uso prévio de antidepressivo $)^{15}$.

A Tabela 3 mostra as principais diferenças observadas entre a depressão unipolar e bipolar ${ }^{16}$. 
Brunoni AR. Transtornos mentais comuns na prática clínica.

TABELA 3. Achados mais comuns na depressão bipolar do que unipolar

\begin{tabular}{ll}
\hline Fenomenologia (Ex. Psíquico) & Resposta ao tratamento com antidepressivos \\
\hline Hipersonia, hiperfagia & "Virada" maníaca ou hipomaníaca \\
\hline Psicose & Piora dos sintomas \\
\hline Sintomas de (hipo)mania concomitantes & Resposta muito rápida \\
\hline Curso Clínico & Resposta aguda, mas não profilática \\
\hline Idade mais precoce & Outros \\
\hline Curso recorrente, múltiplos episódios & Antecedentes Familiares para TAB \\
\hline Pós-parto & Personalidade Hipertímica \\
\hline
\end{tabular}

\section{Tratamento}

O tratamento farmacológico da depressão unipolar foi reavaliado recentemente pelo estudo $\mathrm{STAR}^{*} \mathrm{D}$ (Sequenced Treatment Alternatives to Relieve Depression), um ensaio clínico multicêntrico pragmático em que pacientes recebiam inicialmente um antidepressivo (citalopram) e, caso a resposta não fosse satisfatória, esse antidepressivo era substituído por um outro ou se associava um medicamento ao já prescrito ${ }^{17}$.

Este estudo não conseguiu detectar um antidepressivo que fosse superior aos outros, mas demonstrou que (1) o tratamento do Transtorno Depressivo Maior é possível na atenção primária e em ambulatórios de clínica médica por médicos clínicos; (2) caso haja resposta (quando o paciente descreve uma melhora por volta de $50 \%$ dos sintomas) porém, não remissão (quando não há mais sintomas depressivos), deve-se aumentar a dose do antidepressivo; (3) deve-se esperar oito semanas antes de decretar a falência terapêutica; (4) caso haja falha terapêutica, pode-se tanto optar por associar quanto por trocar para um segundo antidepressivo, pois ambas as opções apresentam respostas semeIhantes, e; (5) após duas tentativas mal-sucedidas, as taxas de resposta estabilizam em torno de $60 \%$; nesses casos, é prudente encaminhar o paciente a um especialista ${ }^{17}$. Os principais antidepressivos estão listados na Tabela 4.

TABELA 4. Principais antidepressivos utilizados na prática clínica

\begin{tabular}{|c|c|c|c|}
\hline Antidepressivo & $\begin{array}{l}\text { Dose inicial } \\
\text { (mg/dia) }\end{array}$ & $\begin{array}{c}\text { Dose terapêutica - } \\
\text { máxima }\end{array}$ & Efeitos colaterais \\
\hline \multicolumn{4}{|l|}{ Tricíclicos } \\
\hline Amitriptilina & 25 & $75-300$ & \multirow{4}{*}{$\begin{array}{l}\text { Sedação, hipotensão postural, ganho de peso, arritmias, boca } \\
\text { seca, visão turva, retenção urinária, prisão de ventre e dis- } \\
\text { função sexual. Muitas interações medicamentosas. Risco de } \\
\text { overdose. }\end{array}$} \\
\hline Imipramina & 25 & $50-300$ & \\
\hline Nortriptilina & 25 & $50-200$ & \\
\hline Clomipramina & 25 & $75-300$ & \\
\hline \multicolumn{4}{|c|}{ Inibidores seletivos da recaptura de serotonina (ISRS) } \\
\hline Fluoxetina & 20 & $20-80$ & \multirow{4}{*}{$\begin{array}{l}\text { Insônia, agitação, sedação, efeitos gastro-intestinais, disfunção } \\
\text { sexual. Menos interações e menor risco de overdose que os } \\
\text { tricíclicos. }\end{array}$} \\
\hline Paroxetina & 20 & $20-80$ & \\
\hline Sertralina & 50 & $50-300$ & \\
\hline Citalopram & 20 & $20-80$ & \\
\hline \multicolumn{4}{|l|}{ Inibidores da MAO } \\
\hline Tranilcipromina & 20 & $20-60$ & \multirow{3}{*}{$\begin{array}{l}\text { Usados na depressão refratária. Restrição alimentar para } \\
\text { alimentos com tiramina (queijo, vinho, enlatados) pelo risco de } \\
\text { crise hipertensiva. }\end{array}$} \\
\hline Fenelzina & 30 & $30-90$ & \\
\hline Moclobemida & 300 & $300-600$ & \\
\hline
\end{tabular}

Por fim, diversas meta-análises recentes mostraram a eficácia do tratamento psicoterápico nos Transtornos Depressivos Maiores. Apesar de a combinação psicoterapia/farmacoterapia ser ideal, em alguns casos (depressões leves e moderadas, a preferência do paciente, recusa em usar antide- 
pressivos) a psicoterapia pode ser aplicada isoladamente no tratamento dos Transtornos Depressivos Maiores $^{18,19}$.

\section{Transtorno Afetivo Bipolar}

O Transtorno Afetivo Bipolar caracteriza-se por episódios depressivos alternando com episódios maníacos (TAB I) ou hipomaníacos (TAB II). Enquanto o TAB I acomete na mesma proporção homens e mulheres, o TAB II é mais comum em mulheres. A idade média de início do quadro é em torno de 20 anos. O diagnóstico de TAB I é feito apenas com um episódio de mania. Para o TAB II, é necessário que haja pelo menos um episódio de hipomania junto com um episódio prévio de Transtorno Depressivo Maior. Mania é definida como humor elevado, expansivo ou irritado por uma semana com mais três sintomas auxiliares. A hipomania também apresenta um humor elevado ou irritado, mas de menor intensidade que a mania, e também necessita de três sintomas auxiliares por, no mínimo, quatro dias ${ }^{9}$. A Tabela 4 mostra os sintomas auxiliares que acompanham os quadros de mania e hipomania.

TABELA 5. Sintomas auxiliares de mania ou hipomania

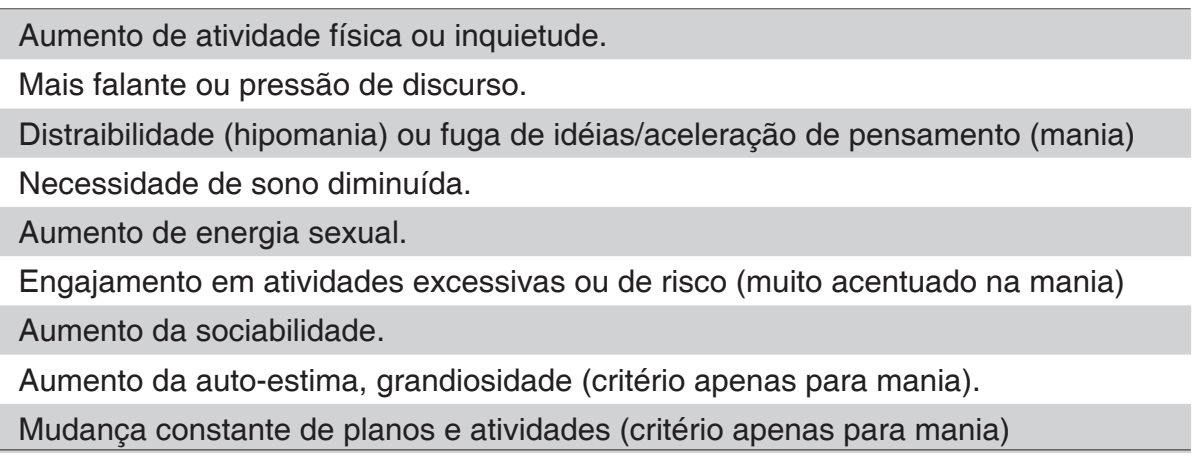

Quando os critérios mais restritos do DSM-IV são aplicados em estudos populacionais, a prevalência de TAB I e TAB II é de cerca de $1 \%$ cada $^{4}$. Contudo, alguns autores propõem que a prevalência na população de $T A B$ pode chegar a $5-10 \%$ e, portanto, ser tão comum quanto o $\mathrm{TDM}^{20}$ - de fato, isto pode ocorrer caso o número de critérios diagnósticos e o tempo de duração para caracterização de um episódio hipomaníaco ou maníaco sejam menores. Por outro lado, ainda é controverso se as formas mais brandas de "bipolaridade" diagnosticadas por estes critérios devem ser tratadas da mesma forma que os quadros mais graves que preenchem os critérios diagnósticos "clássicos" do DSM-IV.
O tratamento do Transtorno Afetivo Bipolar pode ser dividido em: tratamento agudo da mania ou da depressão e tratamento profilático. Para o tratamento agudo da mania (ou seja, episódio maníaco agudo) costuma-se utilizar lítio em monoterapia ou associado com antipsicótico ${ }^{21}$. Para o tratamento agudo da depressão (ou seja, episódio depressivo maior em paciente com TAB), utiliza-se lítio em monoterapia ou associado a antidepressivo, eletroconvulsoterapia (ECT) ou inibidores da monoamino-oxidase (iMAO) ${ }^{22}$. Por fim, para a profilaxia de novos episódios pode-se utilizar lítio, lamotrigina, olanzapina, carbamazepina ou divalproato de sódio (Tabela 6).

TABELA 6. Principais estabilizadores de humor usados na prática clínica

\begin{tabular}{|c|c|c|c|c|}
\hline Droga & Dose terapêutica $(\mathrm{mg} / \mathrm{d})$ & Efeitos colaterais & Anti- Mania & Anti-Depressivo \\
\hline Lítio & Litemia:0,6-12mEq/L & $\begin{array}{l}\text { tremores, ganho de peso, acne, hipo- } \\
\text { tiroidismo }\end{array}$ & +++ & ++ \\
\hline Valproato & $500-2000$ & tremores, ataxia, hepatite, DRGE & +++ & + \\
\hline Carbamazepina & $400-1200$ & tremores, fadiga, rash, hiponatremia & +++ & - \\
\hline Lamotrigina & $25-300$ & $\begin{array}{l}\text { rash, Síndrome de Steven-Johnson } \\
\text { (rara) }\end{array}$ & - & +++ \\
\hline Olanzapina & $2,5-25$ & ganho de peso (substancial), sedação & +++ & ++ \\
\hline
\end{tabular}


Brunoni AR. Transtornos mentais comuns na prática clínica.

\section{Transtornos de ansiedade}

Os transtornos ansiosos são um grupo heterogêneo de sintomas e comportamentos que, em conjunto, acometem por volta de $10 \%$ da população ${ }^{4,10}$. O sintoma em comum de todos os transtornos ansiosos é a experimentação de ansiedade, que pode ser cognitiva (antecipação negativa e desastrosa de eventos futuros) ou física (sintomas de liberação autonômica como taquicardia, taquipnéia, sudorese fria, dor abdominal, entre outros). Menos comumente, a ansiedade intensa também pode levar à cisão da consciência, desencadeando fenômenos dissociativos - como alucinações, amnésias seletivas e perda de identidade, quase sempre de natureza transitória.

A forma, a duração e os eventos precipitantes que estão relacionados com estas vivências de ansiedade permitem caracterizar algumas síndromes específicas que estão listadas na Tabela 7.

TABELA 7. Sintomas de ansiedade física (hiperatividade autonômica) e ansiedade psíquica

\begin{tabular}{lll}
\hline \multicolumn{2}{c}{ Ansiedade física } & \multicolumn{1}{c}{ Ansiedade psíquica } \\
\hline Palpitação & Angústia & Preocupação excessiva \\
Sudorese & Tontura & Antecipação negativa do futuro \\
Tremores & Parestesias & "Remoer" de pensamentos \\
Falta de ar & Náuseas & Medo excessivo de algo ou alguém \\
\hline Dor torácica & Dor abdominal & Comportamento evitativo/recluso \\
\hline
\end{tabular}

Desta maneira, no Transtorno de Ansiedade Generalizada (TAG), a ansiedade é predominantemente cognitiva, de natureza protraída e crônica, sendo expressa na forma de uma preocupação constante com diversos eventos ou atividades por mais de seis meses. A ansiedade física é referida como excesso de tensão e de fadiga, sensação de esgotamento no final do dia, mas com repouso, geralmente conturbado ou não eficaz, sendo comum apresentar insônia e irritabilidade excessiva. Referir angústia (sensação de sufocamento ou aperto no peito) também é comum ${ }^{10}$.

Nos Ataques de Pânico, a ansiedade se precipita de forma súbita e intensa. O indivíduo experimenta uma sensação inesperada de perigo ou destruição iminente que se acompanha de vários sintomas de hiperatividade autonômica. No Transtorno de Pânico (TP), os ataques de pânico tornam-se freqüentes, o paciente passa a "ter medo de ter medo", ou seja, de ter novos ataques, muitas vezes evitando ficar em locais com grande número de pessoas ou em se distanciar muito de casa (condição denominada Agorafobia) ${ }^{10}$.

A fobia social é uma ansiedade anormal em situações sociais comuns, como conhecer pessoas estranhas, falar em público, namorar, ir a festas ou a encontros sociais ou discordar da opinião alheia. Tais situações são percebidas como ameaçadoras, levando a sintomas de ansiedade física, que são percebidos pelo paciente, o que acaba por reforçar sua sensação de fracasso ou inaptidão social23.

Por fim, o Transtorno Obsessivo-Compulsivo (TOC) é caracterizado por pensamentos obsessivos (ou seja, intrusivos, desagradáveis e que geram ansiedade) e comportamentos compulsivos (ou seja, ritualizados, que buscam aplacar a ansiedade gerada pelas obsessões) levando a significativo prejuízo pessoal e social ${ }^{10}$. Por diversos autores interpretarem que a ansiedade não é o sintoma principal do Transtorno Obsessivo Compulsivo, além de outras evidências neurobiológicas e fenomenológicas que aproximam o Transtorno Obsessivo Compulsivo de outros diagnósticos, como o "Jogo Patológico" (um transtorno do controle do impulso em que há uma necessidade de difícil controle em fazer apostas ou obter dinheiro para jogar) talvez ele seja realocado em uma seção que não a de Transtornos de Ansiedade. Desta maneira, é possível que em edições futuras do DSM e da Classificação Internacional de Doenças o Transtorno Obsessivo Compulsivo não seja mais classificado dentro dos transtornos de ansiedade, mas sim em uma categoria a parte ${ }^{24}$ (Tabela 8 ). 
TABELA 8. Critérios diagnósticos para os diversos tipos de transtornos de ansiedade

\begin{tabular}{|c|c|}
\hline Ataque de pânico & Fobia simples \\
\hline Pelo menos 4 critérios dos listados na Tabela 6. & $\begin{array}{l}\text { Medo excessivo e persistente pela presença ou antecipação } \\
\text { de um objeto ou de uma situação, podendo levar a uma crise } \\
\text { ansiosa. }\end{array}$ \\
\hline Transtorno do PÂNICO & Fobia social \\
\hline $\begin{array}{l}\text { Episódios de Pânico repetitivos, inesperados, com } \\
\text { comprometimento importante da qualidade de } \\
\text { vida. }\end{array}$ & $\begin{array}{l}\text { Medo excessivo e persistente de situações sociais em que a } \\
\text { pessoa seja exposta a estranhos ou a julgamento, havendo o te- } \\
\text { mor de passar por algo humilhante, podendo levar a um ataque } \\
\text { de pânico. }\end{array}$ \\
\hline Agorafobia & Transtorno de ansiedade generalizada \\
\hline $\begin{array}{l}\text { Ansiedade em estar em lugares de difícil escapa- } \\
\text { tória, o que leva a um comportamento evitativo. }\end{array}$ & $\begin{array}{l}\text { Preocupação e ansiedade excessivas com diversos eventos e } \\
\text { atividades por um período maior de } 6 \text { meses; levando a sin- } \\
\text { tomas como: cansaço, fadiga, irritabilidade, tensão muscular, } \\
\text { insônia e dificuldade em se concentrar. }\end{array}$ \\
\hline Obsessão & Transtorno obsessivo-compulsivo \\
\hline $\begin{array}{l}\text { Pensamentos recorrentes, persistentes, desagra- } \\
\text { dáveis e não facilmente suprimíveis }\end{array}$ & \multirow{3}{*}{$\begin{array}{l}\text { Transtorno caracterizado por pensamentos obsessivos (geral- } \\
\text { mente de violência, morte ou sexo) e comportamentos compul- } \\
\text { sivos neutralizadores (geralmente de limpeza ou organização) } \\
\text { em que há perda significativa da qualidade de vida. Geralmente } \\
\text { o paciente percebe o TOC como algo irracional e excessivo, } \\
\text { porém não controlável. }\end{array}$} \\
\hline Compulsão & \\
\hline $\begin{array}{l}\text { Comportamentos repetitivos com o propósito de } \\
\text { neutralizar uma obsessão }\end{array}$ & \\
\hline
\end{tabular}

\section{Diagnóstico diferencial}

O Transtorno do Pânico faz diagnóstico diferencial com inúmeras condições clínicas em que há hiperatividade autonômica aguda, como em alguns quadros neurológicos (epilepsia de lobo temporal, acidente vascular cerebral), cardíacos (síndromes coronarianas agudas, arritmias) e endócrinos (feocromocitoma, hipertiroidismo). Ainda, o Transtorno de Ansiedade Generalizada e a fobia social podem levar a sintomas de ansiedade aguda parecidos com as condições clínicas já mencionadas havendo necessidade de diagnóstico diferencial com muita frequência.

\section{Tratamento}

De maneira geral, o tratamento dos Transtor- nos de Ansiedade se beneficia com a combinação de terapias farmacológicas e não-farmacológicas ${ }^{9}$. Por exemplo, na fobia social, enquanto o uso de antidepressivos e ansiolíticos pode diminuir os sintomas físicos de ansiedade, a terapia cognitivocomportamental poderá, por meio de terapia de exposição e treinamento de habilidades sociais, ensinar novos comportamentos funcionais e positivos ao paciente ${ }^{25}$.

Vale a pena destacar que a clomipramina é um Inibidor Seletivo da Recaptação da Serotonina tido como de escolha para o tratamento do Transtorno Obsessivo-Compulsivo. Para os demais transtornos de ansiedade, utilizam-se os antidepressivos listados na Tabela 4. Para abortar ou prevenir crises de ansiedade aguda, costuma-se utilizar benzodiazepínicos (alprazolam, clonazepam, lorazepam) e/ou beta-bloqueadores em doses baixas.

TABELA 9. Alguns benzodiazepínicos usados nos Transtornos de Ansiedade

\begin{tabular}{lccccc}
\hline \multicolumn{1}{c}{ Droga } & Dose & Ação & Ansiolítico & Hipnótico & Efeitos colaterais \\
\hline Midazolam & $7,5-15 \mathrm{mg}(\mathrm{VO})$ & Curta & + & +++ & \\
Alprazolam & $0,25-2 \mathrm{mg}(\mathrm{VO})$ & Intermediária & ++ & + & Uso crônico pode levar a \\
Lorazepam & $2 \mathrm{mg}(\mathrm{VO})$ & Intermediária & +++ & ++ & alterações de memória, de \\
Clonazepam & $0,5-2 \mathrm{mg}(\mathrm{VO})$ & Longa & ++ & + & cognição e de concentração. \\
Diazepam & $5-10 \mathrm{mg}(\mathrm{VO})$ & Longa & +++ & ++ & \\
\hline
\end{tabular}


Brunoni AR. Transtornos mentais comuns na prática clínica.

\section{Transtornos somatoformes}

Os transtornos somatoformes (TS) são condições de difícil reconhecimento e tratamento tanto pelo psiquiatra quanto pelo não-psiquiatra. $\mathrm{O}$ Transtorno Somatoforme pode ser entendido no seu sentido mais amplo, que é a manifestação de sintomas físicos de origem exclusivamente psicogênica. Contudo, esta definição é prejudicial, pois pressupõe uma divisão entre "corpo" e "mente", e pode dar uma idéia de que os sintomas físicos são "simulados" ou "controlados" pelo paciente, além de não englobar situações em que há somatização associada com doença "orgânica" e, por outro lado, não distinguir casos em que a somatização é secundária a outros transtornos mentais ${ }^{9}$. Desta maneira, os transtornos somatoformes devem ser diferenciados de outros transtornos mentais associados com sintomas físicos (por exemplo, um paciente com depressão com muitas queixas álgicas) e de transtornos de simulação (em que o paciente produz o sintoma de maneira intencional, consciente e proposital). As síndromes funcionais somáticas se encontram em um "limbo" na nosologia médica: apesar de serem, essencialmente, sintomas físicos sem explicação orgânica, elas se diferenciam dos transtornos somatoformes por constituírem síndromes clínicas típicas. Algumas, contudo, possuem uma duplicidade diagnóstica - a síndrome do intestino irritável, por exemplo, é classificada como F45.3 (Transtorno Somatoforme autonômico) e também em K58 (outras doenças do intestino), pela CID-109.
Ambulatorialmente, o médico clínico deve desconfiar de um paciente somatizador quando ele apresentar diversos sintomas físicos sem explicação biológica plausível, motivando-o mesmo a procurar auxílio médico constante e realização de grande número de exames (comportamento conhecido como doctor shopping). Deve ser ressaltado, contudo, que sintomas de somatização só constituem um transtorno quando há um quadro crônico e repetitivo de somatização que gera um impacto significativo na vida do paciente.

O Transtorno Somatoforme deve ser compreendido levando-se em conta aspectos biográficos e psicossociais do paciente. Por exemplo, uma paciente de meia idade que manifesta e mantém por vários anos diversas queixas cardíacas ("aumento de pressão", taquicardia, dispnéia, etc.) as quais costuma atribuir a um "princípio de infarto", levando-a a buscar auxílio médico e não acreditando que não tem doença cardíaca. Tal paciente preenche critérios para um Transtorno Somatoforme Indiferenciado (preencheria critérios diagnósticos para hipocondria caso referisse sintomas de diversas doenças). Examinando sua história de vida, por exemplo, poderse-ia descobrir que tal paciente passou a ter estes sintomas após ter se divorciado e passado a morar sozinha - desta maneira, o significado destes sintomas poderia estar relacionado à atenção que recebe quando é levada para o hospital por sua família.

Os critérios diagnósticos para os principais transtornos somatoformes estão listados na Tabela 10.

Tabela 10. Principais transtornos somatoformes

\section{Transtorno de somatização}

História de diversas queixas físicas desde a infância e que levaram a comprometimento social e pessoal. As queixas abrangem quatro esferas: dor - 4 sintomas (cefaléia, lombalgia, articulações, dismenorréia, dispareunia, disúria, etc); gastro-intestinal - 2 sintomas (náuseas, eructações, vômitos, diarréia, intolerância alimentar); sexual - 1 sintoma (disfunção erétil, anorgasmia, amenorréia, metrorragia), e; pseudo-neurológico - 1 sintoma (paralisias, alucinações, cegueiras, etc). Os sintomas não são esclarecidos após investigação, tampouco são intencionalmente provocados ou simulados.

\section{Transtorno somatoforme indiferenciado}

Como o transtorno de somatização, porém sem preencher os critérios do número de sintomas físicos.

Transtorno conversivo

Sintomas pseudo-neurológicos (ou seja, autonômicos, motores ou sensoriais que podem ter origem neurológica), que não são esclarecidos após investigação, tampouco são intencionalmente provocados nem simulados.

\section{Transtorno Doloroso}

Sintomas dolorosos, associados ou não com condições clínicas, que não são esclarecidos após investigação, tampouco são intecionalmente provocados nem simulados.

\section{Hipocondria}

Preocupação com medo de ter uma doença por uma interpretação equivocada dos sintomas físicos; preocupação que persiste após avaliação médica e reasseguramento, e que tem curso crônico. 
Tratamento

O tratamento dos Transtornos Somatoformes deve envolver um clínico e um psiquiatra. Ambos os profissionais devem formar uma verdadeira aliança terapêutica com o paciente, para conseguir sua confiança. A relação médico-paciente com o paciente com Trnastorno Somatoforme deve envolver: atenção ao paciente (ouvir suas queixas, suas explicações) e não aos seus exames; aceitação incondicional (não rejeitar ou encaminhar o paciente, ter paciência e não se precipitar em buscar doenças que expliquem o quadro). A expectativa em relação ao tratamento deve ser realista: apesar do transtorno conversivo remitir completamente na maioria dos casos, a hipocondria, o transtorno de somatização e os transtornos dolorosos tem um curso crônico, com muitas recaídas e de tratamento difícil26.

O tratamento com o psiquiatra envolverá farmacoterapia (tratamento das condições mentais subjacentes) e, eventualmente, técnicas cognitivocomportamentais. Ao clínico (e também ao psiquiatra) caberá tranquilizar o paciente em relação a suas queixas, o que envolve: ouvir pacientemente todas as queixas do paciente e investigá-las por meio de perguntas (não de exames), validar as queixas (não dizer que "é da cabeça", mas sim que os sintomas são reais e estimular o próprio paciente a buscar hipóteses não-médicas para essas doenças) e estar atento a novos sintomas, investigando-os caso haja necessidade.

\section{Quando encaminhar um paciente para o psiquiatra?}

Como discutido neste artigo, em muitas situações o médico clínico pode tratar adequadamente os principais transtornos mentais. Em algumas situações, contudo, será necessário encaminhar alguns pacientes para o psiquiatra. Além de transtornos mais raros e não mencionados neste artigo (como Esquizofrenia, Transtornos Alimentares ou dependências químicas), outras condições que podem exigir um encaminhamento são: falha terapêutica de pelo menos dois tratamentos farmacológicos para algum dos diagnósticos discutidos, sintomas ou co-morbidades psiquiátricas de alto risco ou de difícil tratamento (por exemplo, ideação suicida recorrente, comportamentos impulsivos, deficiência mental), ou ainda, caso o paciente apresente tantas co-morbidades clínicas que não haja tempo adequado para o tratamento conjuntamente do transtorno psiquiátrico ${ }^{25}$.

TABELA 11. Indicações para encaminhamento para um psiquiatra

Transtornos psiquiátricos de difícil tratamento: transtornos somatoformes, transtornos alimentares, transão os mais sinceros votos, tornos relacionados ao uso de substâncias, entre outros.

Falha terapêutica de pelo menos dois tratamentos instituídos.

Casos de difícil manejo: Transtorno de personalidade, deficiência mental, ideação suicida recorrente.

Múltiplas co-morbidades clínicas, necessitando de acompanhamento conjunto.

\section{Casos clínicos}

Caso clínico 1: Um paciente de 36 anos é levado ao pronto-socorro por ter fraturado o punho em uma briga de bar. Apresenta-se agitado, irritado e falando alto. Quando questionado sobre o motivo da briga, não consegue esclarecer o motivo, contudo, rapidamente declara que já transou com mais de 50 mulheres e que não dorme há dois dias. Relata que é do interior do Estado e que saiu de casa há duas semanas, deixando esposa e filhos, para "levar uma vida de aventuras". Nega uso de substâncias ou comorbidades clínicas.

1) Os diagnósticos de síndrome e de transtorno deste paciente são:

a) síndrome maniforme, transforno afetivo bipolar;

b) síndrome maniforme, transtorno afetivo unipolar maníaco;

c) síndrome mista, transtorno afetivo bipolar;

d) síndrome ansiosa, transtorno de ansiedade generalizada;

e) síndrome ansiosa, transtorno afetivo bipolar.

Resposta: (A) - O paciente apresenta critérios para diagnóstico de mania (apresenta há mais de duas semanas humor irritado, grandiosidade, engajamento de atividades de risco, reduzida necessidade de sono, pressão de discurso). Apenas um episódio de mania é suficiente para o diagnóstico de Transtorno Afetivo Bipolar.

2) O tratamento agudo e de manutenção para este paciente é: 
a) lítio associado à fluoxetina (agudo); Lítio (manutenção);

b) haloperidol (agudo); apenas acompanhamento clínico (manutenção);

c) Iamotrigina (agudo); lamotrigina (manutenção);

d) lítio + haloperidol (agudo); Lítio (manutenção);

e) lítio + lamotrigina (agudo); lamotrigina (manutenção).

Resposta: (D) - O tratamento da mania aguda deve ser feito com um estabilizador de humor, sendo geralmente associado um anti-psicótico. Na fase de manutenção utiliza-se apenas um estabilizador de humor. Apesar de lamotrigina ser um estabilizador de humor, ela não é indicada em casos de mania aguda.

Caso clínico 2: Paciente de 21 anos, estudante universitária, procurou o pronto socorro com sensação de palpitação, dispnéia, sudorese fria e tremores. Apresentava-se bastante nervosa e emocionalmente aflita. Conta que apresentou subitamente palpitações durante o treino de voleibol. Relata que estes episódios ocorreram outras três vezes no último mês, sempre começando com palpitações de início súbito - quando ia visitar uma amiga, após brigar com o namorado e na fila do banco. Relata que está "passando por alguns problemas" recentemente. Exame físico: Freqüência Cardíaca de 102 batimentos por minuto, rítmica, sem outras alterações.

3) Marque o principal diagnóstico diferencial e o procedimento diagnóstico indicado:

a) ataque de pânico - Diazepam 10mg por via oral;

b) transtorno do pânico - Diazepam 5mg por via endovenosa;

c) arritmia cardíaca - eletrocardiograma;

d) transtorno de somatização - dar alta imediatamente à paciente, para não valorizar suas queixas;

e) hipertireoidismo - dosagem de TSH (tireotropina) e T4-livre (tiroxina livre).

Resposta: (C) Trata-se de uma paciente jovem, de sexo feminino, com conflitos pessoais e apresentando quatro episódios compatíveis com ataques de pânico, sugerindo, portanto, um diagnóstico de Transtorno do Pânico. Contudo, a paciente apresenta tais sintomas há menos de um mês e sempre refere palpitações. Este é um diagnóstico de exclusão, devendo primeiramente descartar-se outras causas, como arritmias malignas.

4) No tratamento do transtorno do pânico os medicamentos mais utilizados são: a) inibidores seletivos da recaptação de serotonina (ISRS), benzodiazepínicos e betabloqueadores;

b) benzodiazepínicos, inibidores da monoaminooxidase e amiodarona;

c) inibidores seletivos da recaptação de serotonina, amiodarona e formoterol;

d) antidepressivos tricíclicos, procainamida e salbutamol;

e) antidepressivos tricíclicos, lamotrigina e betabloqueadores.

Resposta: (A) - todas as medicações listadas nessa alternativa podem ser utilizadas no tratamento do Transtorno do Pânico - a escolha deve ser baseada no perfil de efeitos colaterais de cada droga mais adequado à paciente.

Caso clínico 3: Paciente de 33 anos, procura clínico geral por se sentir muito deprimida há seis meses. Refere muita fadiga, cansaço e indisposição, com muito sono e ganho de peso de $7 \mathrm{~kg}$ no período. Nega pensamentos de morte, de culpa ou de menos-valia. Conta também que já usou fluoxetina, sertralina, paroxetina e nortriptilina por episódios depressivos no passado $-01^{\circ} \mathrm{com} 18$ anos, o $2^{\circ} \mathrm{com} 22$ anos, o $3^{\circ} \mathrm{com} 23$ anos e o $4^{\circ}$ após o nascimento de seu filho, com 28 anos. "A diferença", conta, "é que antes a depressão passava rápido - agora está demorando!". Confessa que tentou usar fluoxetina no episódio atual, sem prescrição médica, mas que ficou mais irritada, ansiosa, agitada e sem sono. Nos períodos em que não está deprimida, sente-se muito bem, bastante ativa, cheia de energia e dorme pouco - cerca de seis horas por noite.

5) Qual o diagnóstico mais provável da paciente?

a) transtorno depressivo maior.

b) distimia.

c) transtorno Afetivo Bipolar, episódio atual depressivo.

d) transtorno de Personalidade Borderline.

e) transtorno de ansiedade generalizada.

Resposta: (C) - Transtorno Afetivo Bipolar, episódio atual depressivo - a paciente apresenta várias características sugestivas de uma depressão bipolar: depressão pós-parto, idade precoce de início, resposta paradoxal a antidepressivos, fenomenologia da depressão com sintomas vegetativos reversos (hipersonia, ganho de peso), personalidade hipertímica basal.

6) Considerando o diagnóstico mais provável da paciente, qual é o dado de história menos provável? 
a) descrição, por parte de conhecidos, de episódios de hipomania curtos ao longo da vida.

b) antecedentes familiares de $1^{\circ} \mathrm{grau}$ com TAB.

C) descrição, por parte de conhecidos, de episódios de depressão breve, não tratados, ao longo da vida.

d) uma percepção clara, por parte da paciente, de seus episódios de hipomania.

e) período ou períodos da vida em que houve envolvimento em atividades de risco (uso de substâncias ilícitas ou relações sexuais desprotegidas, por exemplo)

Resposta: (D) Pacientes bipolares podem ter dificuldade em reconhecer seus períodos de hipomania, especialmente durante um episódio depressivo. Por isso é necessário questionar os familiares e buscar outros indícios que possam corroborar o diagnóstico, como as alternativas (A), (C) e (E). Antecedentes familiares de TAB (alternativa $B$ ) é bastante sugestivo de TAB.

Caso clínico 4: Paciente do sexo masculino, 45 anos encaminhado ao Hospital das Clínicas, pois nenhum outro médico "descobriu" a sua doença. Traz uma sacola de exames, em que se encontram: tomografia computadorizada de crânio, endoscopia digestiva alta, colonoscopia, eletroneuromiografia e diversos exames de sangue. Nenhum dos exames apresenta alterações importantes. Ainda, traz relatos de três internações hospitalares breves para investigação de diarréia crônica, desmaios com perda de consciência rápida e uma apendicectomia "branca" (o anatomopatológico era normal). Na infância, frequentemente ia ao pronto-socorro por crises de asma. No momento, queixa-se de formigamento nas mãos e dores na cabeça e pede para realizar uma ressonância nuclear magnética de crânio, pois outro médico lhe havia dito que "seu problema era na cabeça".

7) Assinale o diagnóstico mais provável:

a) transtorno de somatização;

b) hipocondria;

c) transtorno doloroso;

d) transtorno conversivo;

e) transtorno depressivo.

Resposta: (A) - Transtorno de Somatização. O paciente apresenta, desde a infância, diversas queixas físicas não esclarecidas após investigação e um padrão de procura por auxilio médico, realizando diversos exames ("doctor shopping"). Não apresenta hipocondria (alternativa B) porque suas queixas não são focadas em doenças, mas em sintomas.
Alternativas C e D: Apesar de o paciente apresentar um sintoma conversivo (perda de consciência) e um sintoma doloroso (cefaléia) o quadro predominante é a multiplicidade dos sintomas. Por fim, apesar de o transtorno depressivo ser uma co-morbidade comum do transtorno de somatização, ainda não está caracterizado este diagnóstico no paciente (alternativa E).

8) Assinale a melhor conduta:

a) encaminhar ao neurologista e dar alta;

b) orientar a não marcar nova consulta com clínico, encaminhar ao psiquiatra, e dar alta do ambulatório de clínica médica;

c) pedir uma ressonância nuclear magnética (RNM) e marcar retorno em seis meses, porque a RNM demora para ficar pronta;

d) questionar sobre a correlação dos sintomas com sua história de vida, investigar quadro depressivo associado e marcar retorno em duas semanas;

e) repetir novamente todos os exames para provar para o paciente que ele não tem doença física.

Resposta: (D) No tratamento do paciente com Transtorno de Somatização é fundamental o estabelecimento de uma boa relação médico-paciente. Os retornos devem ser breves e o encaminhamento para especialistas, bem como a propedêutica armada, deve ser feito apenas se absolutamente necessário. A comunicação com o paciente não deve ser feita focada apenas em sintomas e doenças. Apesar de o encaminhamento ao psiquiatra ser necessário, este não deve ser feito até que o paciente crie um bom vinculo com seu médico. A repetição de todos os exames é desnecessária e, mesmo que os exames venham normais, é de pouca valia na condução do caso.

9) Assinale o achado de exame clínico mais compatível com Transtorno Conversivo:

a) diminuição de reflexos nos membros inferiores;

b) parestesia em bota e luva;

c) hemi-anestesia começando exatamente na linha mediana do corpo, por todo o corpo;

d) fasciculações;

e) amaurose (cegueira) súbita.

Resposta: (C) O transtorno conversivo é caracterizado por sintomas pseudo-neurológicos - ou seja, aqueles que não podem ser explicados por doenças neurológicas ou médicas. Desta maneira, doenças musculares, degenerativas ou da junção neuro-muscular podem cursar com fasciculações ou diminuição de reflexos. Parestesia em bota e luva costuma ser observada em pacientes com diabetes melito ou deficiência vitamínica. Diversas causas neuro-oftalmológicas 
podem se apresentar com amaurose (cegueira) súbita, como oclusão da artéria central da retina ou glaucoma agudo. Por outro lado, hemi-anestesia por doença neurológica (por exemplo um acidente vascular cerebral) não se apresenta de forma completa, por todo o corpo, começando exatamente na linha mediana. É a alternativa correta.

10) Paciente de 25 anos, descreve que apresentou nos últimos dois anos várias vezes quadro súbito de medo intenso, com duração de aproximadamente três minutos. Não consegue descrever desencadeantes emocionais ou físicos para esse quadro. Antes do quadro, descreve que vê "pequenos monstros saltando da parede para dentro dos meus olhos". Diz que lembra de ter tido este quadro dos quatro aos nove anos, necessitando usar medicação cujo nome não recorda. Atualmente, apresenta tais crises de medo intenso cerca de três vezes por semana, desde então. Tem ensino fundamental incompleto, trabalhava como faxineira em uma empresa, mas desde que o quadro se agravou está de licença médica remunerada.

Assinale a principal hipótese diagnóstica e seu diagnóstico diferencial:

a) transtorno de somatização e transtorno factício;

b) epilepsia de lobo temporal e transtorno do pânico;

c) transtorno do pânico e transtorno factício;

d) transtorno do pânico e esquizofrenia;

e) transtorno factício e esquizofrenia.

Resposta: (B) - A principal hipótese diagnóstica é Epilepsia de Lobo Temporal (ELT), com sintomas psíquicos. Apesar de o quadro lembrar um ataque de pânico, chama a atenção a periodicidade do quadro e a presença de fenômenos alucinatórios. Ainda, a paciente apresenta um curso típico da doença, com um quadro na infância que não se manifesta na $2^{a}$ década de vida e depois volta a apresentar sintomas.

Brunoni AR. Common mental disorders in clinical practice. Rev Med (São Paulo). 2008 out.dez.;87(4):251-63.

ABSTRACT: Background: The prevalence of mental disorders in the general population and primary care is high, placing general practitioners as the main gatekeepers for identification, screening, treatment and referral of patients with psychiatric disorders. Therefore, knowing such conditions is mandatory to decrease the morbidity of mental disorders. Purpose: The aim of this article is to review the main issues related to three prevalent mental conditions: mood disorders, anxiety disorders and somatoform disorders. Results: Primary care physicians should screen for depressive disorders (life-time prevalence: 17\%) by asking on depressive mood and anhedonia. Bipolar disorders (3-5\%) should be ruled out before diagnosing unipolar depressive disorder, as misdiagnosis can increase morbidity. Mood disorders, as well as anxiety disorders $(8 \%)$, can be safely managed today with several antidepressant drugs. On the other hand, somatoform disorders $(6 \%)$ require a combined physician-psychiatrist follow-up - in such cases, the physician should recognize the disorder as well as rule out organic diseases. Conclusion: General practitioners can and should manage common psychiatric disorders in their practice; however, they should also be able to recognize more severe conditions whose management should be taken by a psychiatrist.

KEY WORDS: Mental disorders. Bipolar disorder. Somatoform disorders. Mood disorders.

\section{REFERÊNCIAS}

1. Demyttenaere K, Bruffaerts R, Posada-Villa J, Gasquet I, Kovess V, Lepine JP, et al. Prevalence, severity, and unmet need for treatment of mental disorders in the World Health Organization World Mental Health Surveys. JAMA. 2004;291(21):2581-90.

2. Almeida-Filho $\mathrm{N}$, Mari $\mathrm{J}$ de $\mathrm{J}$, Coutinho $\mathrm{E}$, Franca JF, Fernandes J, Andreoli SB, et al. Brazilian multicentric study of psychiatric morbidity. Methodological features and prevalence estimates. Br J Psychiatry. 1997; 171:524-9.
3. Vorcaro CM, Lima-Costa MF, Barreto SM, Uchoa E. Unexpected high prevalence of 1-month depression in a small Brazilian community: the Bambui Study. Acta Psychiatr Scand. 2001;104(4):257-63.

4. Andrade L, Walters EE, Gentil V, Laurenti R. Prevalence of ICD-10 mental disorders in a catchment area in the city of Sao Paulo, Brazil. Soc Psychiatry Psychiatr Epidemiol. 2002;37(7):316-25.

5. Murray CJ, Lopez AD. Evidence-based health policy- 
lessons from the Global Burden of Disease Study. Science. 1996;274(5288):740-3.

6. Halfin A. Depression: the benefits of early and appropriate treatment. Am J Manag Care. 2007;13(4 Suppl):S92-7.

7. Tesar GE. Should primary care physicians screen for depression? Cleve Clin J Med. 2003;70(6):488-90.

8. Borus JF, Howes MJ, Devins NP, Rosenberg R, Livingston WW. Primary health care providers' recognition and diagnosis of mental disorders in their patients. Gen Hosp Psychiatry. 1988;10(5):317-21.

9. Elkis H, Louza M. Psiquiatria básica. 2nd ed. São Paulo: Atheneu; 2007.

10. American Psychiatric Association. Diagnostic and statistical manual of mental disorders DSM-IV-TR. 4th ed. Arlington, Va: APA; 2000.

11. Pignone MP, Gaynes BN, Rushton JL, Burchell CM, Orleans CT, Mulrow CD, et al. Screening for depression in adults: a summary of the evidence for the U.S. Preventive Services Task Force. Ann Intern Med. 2002;136(10):765-76.

12. USPTF. Screening for depression: recommendations and rationale. Ann Intern Med. 2002;136(10):760-4.

13. Muzina DJ, Colangelo E, Manning JS, Calabrese JR. Differentiating bipolar disorder from depression in primary care. Cleve Clin J Med. 2007;74(2):89, 92, 5-9 passim.

14. Judd LL, Akiskal HS, Schettler PJ, Endicott J, Maser J, Solomon DA, et al. The long-term natural history of the weekly symptomatic status of bipolar I disorder. Arch Gen Psychiatry. 2002;59(6):530-7.

15. Muzina DJ, Calabrese JR. Recent placebo-controlled acute trials in bipolar depression: focus on methodology. Int J Neuropsychopharmacol. 2003;6(3):285-91.

16. El-Mallakh RS, Ghaemi NS. Depressão bipolar: um guia abrangente. Porto Alegre: Artmed; 2008.

17. Gaynes BN, Rush AJ, Trivedi MH, Wisniewski SR, Spencer D, Fava M. The STAR*D study: treating depression in the real world. Cleve Clin J Med. 2008;75(1):57-66.

18. Cuijpers $P$, van Straten $A$, van Oppen $P$, Andersson G. Are psychological and pharmacologic interventions equally effective in the treatment of adult depressive disorders? A meta-analysis of comparative studies. J Clin Psychiatry. 2008;69(11):1675-85; quiz 1839-41.

19. Leichsenring F, Rabung S. Effectiveness of long-term psychodynamic psychotherapy: a meta-analysis. JAMA. 2008;300(13):1551-65.

20. Angst J. Bipolar disorder--methodological problems and future perspectives. Dialogues Clin Neurosci. 2008;10(2):129-39.

21. Bauer MS, Mitchner L. What is a "mood stabilizer"? An evidence-based response. Am J Psychiatry. 2004;161(1):3-18.

22. Fountoulakis KN, Vieta E, Sanchez-Moreno J, Kaprinis SG, Goikolea JM, Kaprinis GS. Treatment guidelines for bipolar disorder: a critical review. J Affect Disord. 2005;86(1):1-10.

23. Muzina DJ, El-Sayegh S. Recognizing and treating social anxiety disorder. Cleve Clin J Med. 2001;68(7):649-57.

24. Bartz JA, Hollander E. Is obsessive-compulsive disorder an anxiety disorder? Prog Neuropsychopharmacol Biol Psychiatry. 2006;30(3):338-52.

25. Bea SM, Tesar GE. A primer on referring patients for psychotherapy. Cleve Clin J Med. 2002;69(2):113-4, 117-8, 120-2, 125-7.

26. Sadock BJ, Sadock VA. Kaplan \& Sadock's: comprehensive textbook of psychiatry. 8th ed. Baltimore: Lippincott Williams \& Williams; 2005. 